\title{
Thermal performance of nanoencapsulated phase change material in high molecular weight polystyrene
}

\author{
Tamara Agner $^{1}$ (D), Amadeo Zimermann ${ }^{1}$ (D), Fabricio Machado² (D), Brenno Amaro da Silveira Neto ${ }^{3}$ (D), \\ Pedro Henrique Hermes de Araújo ${ }^{1}$ (D) and Claudia Sayer ${ }^{1 *}$ (i)
}

\begin{abstract}
'Laboratório de Controle e Processos de Polimerização - LCP, Departamento de Engenharia Química e Engenharia de Alimentos - EQA, Universidade Federal de Santa Catarina - UFSC, Florianópolis, SC, Brasil
\end{abstract}

${ }^{2}$ Laboratório de Desenvolvimento de Processos Químicos - LDPQ, Instituto de Química - IQ, Universidade de Brasília - UnB, Brasilia, DF, Brasil

${ }^{3}$ Laboratório de Química Medicinal e Tecnológica - LaQuiMeT, Instituto de Química - IQ, Universidade de Brasilia - UnB, Brasília, DF, Brasil

${ }^{\dagger}$ Selected paper presented at the 15th Brazilian Polymer Conference - (15thCBPol) held in Bento Gonçalves, Brazil, on 27-31 October, 2019.

*claudia.sayer@ufsc.br

\begin{abstract}
A novel nanoencapsulation of n-hexadecane in high molecular weight polystyrene nanoparticles for thermal energy storage was carried out by miniemulsion polymerization using an iron-containing imidazolium-based ionic liquid (IL) as catalyst. The particle size, morphology, molecular weight, and thermal performance of nanoparticles containing the phase change material (PCM) were measured by dynamic light scattering, transmission electron microscopy, gel permeation chromatography, and differential scanning calorimetry, respectively. The nanoparticles were regular spherical, with narrow size distribution and particle size ranged from $138 \mathrm{~nm}$ to $158 \mathrm{~nm}$. The enthalpy of melting for the nanoencapsulated PCM increased from $19 \mathrm{~J} / \mathrm{g}$ to $72 \mathrm{~J} / \mathrm{g}$, as the content of n-hexadecane used increased from $20 \mathrm{wt} \%$ to $50 \mathrm{wt} \%$. In addition, the nanoparticles showed thermal reversibility after 100 thermal cycles. The high molecular weights of the polymer, up to $1800 \mathrm{kDa}$, that could be reached with this IL may have contributed positively to this thermal behavior.
\end{abstract}

Keywords: cationic miniemulsion polymerization, ionic liquid, nanoencapsulation, phase change material.

How to cite: Agner, T., Zimermann, A., Machado, F., Neto, B. A. D., Araújo, P. H. H., \& Sayer, C. (2020). Thermal performance of nanoencapsulated phase change material in high molecular weight polystyrene. Polímeros: Ciência e Tecnologia, 30(2), e2020013. https://doi.org/10.1590/0104-1428.01320

\section{Introduction}

The tireless search for new technologies to capture and store energy in more efficient, ecologically friendly, and low-cost ways has been boosting the development of phase change materials. Latent heat storage materials or simply phase change materials (PCMs) are capable of storing and releasing large amounts of energy during melting and solidification at specific temperature ${ }^{[1]}$. Due to its useful properties, such a higher energy storage densities, and phase change behaviors at almost constant temperature ${ }^{[2]}$ they are widely developed for several applications especially for thermal comfort building, solar heating system, thermal protection, air-conditioning, thermal regulated textiles, electronic devices, among others. ${ }^{[3]}$

Phase change materials are classified into two main categories, organic and inorganic. Most of the organic PCMs have low thermal conductivity and poor thermal response as well as being flammable, however, these limitations may be overcome by employing encapsulation technologies ${ }^{[1]}$. The encapsulation provides protection of PCM from external environmental influences, increases the surface of heat transfer, and the capsule shell helps to retain the changes in the volume that result from the phase change ${ }^{[4]}$.

Several techniques have been used to encapsulate PCMs such as in-situ polymerization which includes miniemulsion polymerization, complex coacervation, sol-gel methods, and solvent extraction/evaporation method, among others. ${ }^{[1]}$ Miniemulsion polymerization is a versatile technique for the in-situ encapsulation of different compounds into polymeric nanoparticles. In general, miniemulsions consist of small $(50-500 \mathrm{~nm})^{[5]}$, stable, and narrowly distributed monomer droplets in a continuous phase ${ }^{[6]}$. The miniemulsion design involves the manipulation of the monomer-water interface stability and the dispersion mechanism (e.g., ultrasonic homogenizers ${ }^{[7-9]}$, rotor-stator systems ${ }^{[7,9]}$, high-pressure homogenizers ${ }^{[8,10]}$, static mixers ${ }^{[7,11]}$, or membranes $\left.{ }^{[9]}\right)$. The high stability of the droplets is ensured by the combination of the surfactant, and the costabilizer, which retards the droplet diffusional degradation (Ostwald ripening). Typically, 
highly hydrophobic and low molecular weight compounds, such as hexadecane, are used as costabilizer. The monomer nanodroplets become the polymerization locus, and in an idealized concept each of these nanodroplets can be regarded as a nanoreactor, without major secondary nucleation or monomer mass transport through the aqueous phase ${ }^{[5]}$. Then, different compounds such as PCMs can be added into the monomer droplets before the miniemulsification step in the water phase, and followed by the polymerization leading to high encapsulation efficiencies.

Luo and Zhou ${ }^{[12]}$ employed the miniemulsion polymerization process to nanoencapsulate a paraffin $\left(T_{\mathrm{m}}=52^{\circ} \mathrm{C}\right)$ using a styrene-methacrylic acid copolymer. They noticed that the amount and type of surfactant, the concentration of comonomer (methacrylic acid), and the monomer/paraffin ratio, as well as the amount of the chain-transfer agent, and the nucleation mechanism did have a significant influence on the process.

de Cortazar and Rodrígue ${ }^{[13]}$ obtained stable nanocapsules containing up to $60 \mathrm{wt} \%$ of paraffin $\left(T_{\mathrm{m}}=60{ }^{\circ} \mathrm{C}\right)$ by miniemulsion polymerization. The droplet nucleation was the main nucleation mechanism for the systems containing under $20 \mathrm{wt} \%$ of paraffin, for higher paraffin contents $(40-60 \mathrm{wt} \%)$ secondary unwanted nucleation mechanism took place. Whereas systems with even higher paraffin concentration $(80 \mathrm{wt} \%$ ) showed phase separation.

Fang et al. published a series of works in which the encapsulation of n-octadecane ${ }^{[14,15]}\left(\Delta H_{\mathrm{m}}=232.3 \mathrm{~J} / \mathrm{g}\right)$, n-tetradecane ${ }^{[2]}\left(\Delta H_{\mathrm{m}}=220.6 \mathrm{~J} / \mathrm{g}\right)$ or $\mathrm{n}$-dotriacontane $\mathrm{e}^{[16]}$ $\left(\Delta H_{\mathrm{m}}=286 \mathrm{~J} / \mathrm{g}\right)$ in polystyrene nanoparticles $(100-170 \mathrm{~nm})$ via miniemulsion polymerization was evaluated. They used a monomer mixture of styrene and up to $2 \mathrm{wt} \%$ of acrylate (butyl, acrylic, or ethyl) to prepare the polystyrene shells. The latent heat of melting of nanoencapsulated PCM was $124.4 \mathrm{~J} / \mathrm{g}$ for $\mathrm{n}$-octadecane, $98.71 \mathrm{~J} / \mathrm{g}$ for $\mathrm{n}$-tetradecane, and $174.8 \mathrm{~J} / \mathrm{g}$ for $\mathrm{n}$-dotriacontane.

Fuensanta et al. ${ }^{[17]}$ encapsulated a paraffin type phase change material $(5-20 \mathrm{wt} \%)$, RT80 $\left(T_{\mathrm{m}}=85^{\circ} \mathrm{C}\right.$, $\Delta H_{\mathrm{m}}=161.1 \mathrm{~J} / \mathrm{g}$ ) in a styrene-butyl acrylate copolymer shell. The particle sizes ranged from $52 \mathrm{~nm}$ to $112 \mathrm{~nm}$ with encapsulation efficiencies close to $80 \%$, and good thermal reversibility after 200 thermal cycles. The thermal energy storage capacity of the nanoparticles was measured by DSC, obtaining melting and crystallization enthalpies in the range of $10-20 \mathrm{~J} / \mathrm{g}$.

Chen et al. produced nanocapsules $(90-200 \mathrm{~nm})$ containing n-dodecanol $\left(T_{m}=27^{\circ} \mathrm{C}, \Delta H_{\mathrm{m}}=222-248 \mathrm{~J} / \mathrm{g}\right)$ with poly(methyl methacrylate) (PMMA) $)^{[18]}$ or styrene-butyl acrylate copolymer ${ }^{[19]}$ as the shell materials by miniemulsion polymerization. The latent heat of nanocapsules was $98.8 \mathrm{~J} / \mathrm{g}$ at $18.2^{\circ} \mathrm{C}$ for the $\mathrm{n}$-dodecanol/PMMA system and $109.2 \mathrm{~J} / \mathrm{g}$ at $18.4^{\circ} \mathrm{C}$ for the n-dodecanol /styrene-butyl acrylate system.

Many types of polymerization reaction mechanisms can be carried out in miniemulsion. Radical polymerization is the most common mechanism, but non-radical polymerization such as ionic polymerization, anionic ${ }^{[20-22]}$ and cationic ${ }^{[23-26]}$, can also be adapted to be carried out in miniemulsion.

Recently, Alves et al. ${ }^{[26]}$ showed that the ironcontaining imidazolium-based ionic liquid 1-n-butyl-3- methylimidazolium heptachlorodiferrate $\left(\mathrm{BMI} \mathrm{Fe}_{2} \mathrm{Cl}_{7}\right)$, proposed by Rodrigues et al. ${ }^{[27]}$ as a new class of cationic catalysts for the styrene polymerization, is water tolerant for the miniemulsion polymerization. BMI. $\mathrm{Fe}_{2} \mathrm{Cl}_{7}$ not only proved to be an efficient initiator in aqueous medium as well as provided the synthesis of polystyrene nanoparticles $(150-175 \mathrm{~nm})$ of high molecular weights (Mv up to $2220 \mathrm{kDa})$, higher than those obtained with the commonly used initiators.

Combining the high molecular weights obtained in the cationic miniemulsion polymerization of styrene catalyzed by the ionic liquid $\mathrm{BMI} . \mathrm{Fe}_{2} \mathrm{Cl}_{7}$ and the encapsulation feature that miniemulsion polymerization technique offers, this work aims to study the encapsulation of n-hexadecane as phase change material in high molecular weight polystyrene nanoparticles. It is expected that the $n$-hexadecane combines its properties as hydrophobic agent, retarding the droplet diffusional degradation (Ostwald ripening), and acts simultaneously as a phase change material storing and releasing energy during the melting and solidification process.

\section{Materials and Methods}

\subsection{Materials}

Technical grade monomer styrene (Sty, Innova) used to prepare the polymeric matrix was distilled under reduced pressure before use and stored at $-4{ }^{\circ} \mathrm{C}$. n-Hexadecane (HD, Sigma Aldrich) was used as costabilizer and as phase change material. Hexadecyltrimethylammonium bromide (CTAB, Sigma Aldrich) was used as surfactant. HD and $\mathrm{CTAB}$ were used as received. The ionic liquid 1-n-butyl-3methylimidazolium heptachlorodiferrate $\left(\mathrm{BMI} \mathrm{Fe}_{2} \mathrm{Cl}_{7}\right)$ used as catalyst was synthesized as reported by Rodrigues et al. ${ }^{[27]}$. Deionized water was used in all reactions.

\subsection{Synthesis of nPCM by miniemulsion polymerization}

The nanoparticles containing the phase change material (nPCM) were synthesized by cationic miniemulsion polymerization using the ionic liquid $\mathrm{BMI} . \mathrm{Fe}_{2} \mathrm{Cl}_{7}$ as catalyst according to the following procedures. The surfactant CTAB $(60 \mathrm{mg})$ was dissolved in deionized water $(11 \mathrm{~mL})$. Different HD contents were evaluated, and the organic phase compositions (HD/Sty wt \%/wt \%) were 5/95, 20/80, 30/70, $35 / 65,40 / 60$, and 50/50. Regardless of composition, $3.3 \mathrm{~g}$ of organic phase was used in all reactions. The aqueous phase and the organic phase were magnetically stirred for $20 \mathrm{~min}$ $(150 \mathrm{rpm})$ before being mixed and pre-emulsified under magnetic stirring (1500 rpm) for further $20 \mathrm{~min}$. The coarse emulsion formed was sonicated for $1 \mathrm{~min}$ ( $10 \mathrm{~s}$ on $/ 5 \mathrm{~s}$ off) at $70 \%$ amplitude (Sonic Dismembrator Model 500 - Fisher Scientific, tip probe size of $1 / 2$ in) using an ice-bath to avoid thermal polymerization. Then, an aliquot $(1 \mathrm{~mL})$ of a $\mathrm{BMI} . \mathrm{Fe}_{2} \mathrm{Cl}_{7}$ aqueous solution, used at 1:1000 molar ratio of IL:Sty, was added to the miniemulsion. All the polymerizations were carried out for $8 \mathrm{~h}$ under nitrogen atmosphere at $85^{\circ} \mathrm{C}$. After polymerization, the latex was cooled down to room temperature. Right after the latex was demulsified with ethanol, and filtered to obtain the nanoparticles containing the PCM. The nanoparticles were washed three times with ethanol to remove the unencapsulated n-hexadecane and then were dried at room temperature. 
Reactions were performed in duplicate. The monomer conversion was determined by gravimetry.

\subsection{Characterization and performance of $n P C M$}

\subsubsection{Dynamic Light Scattering (DLS)}

Intensity average sizes of monomer droplets and polymer nanoparticles were determined by Dynamic Light Scattering - DLS (Zetasizer Nano S, Malvern). Miniemulsion samples were diluted before measurement with a water solution saturated with styrene containing an amount of CTAB below the critical micelle concentration. Latex samples were diluted with deionized water.

\subsubsection{Transmission Electron Microscopy (TEM)}

Nanoparticles morphology was observed by Transmission Electron Microscopy with a JEM-1011 TEM (Jeol) electron microscope operating at an accelerating voltage of $100 \mathrm{kV}$. The diluted latex $(0.01 \mathrm{wt} \%)$ was mounted on a carbon-coated copper grid and was left to dry at room temperature before analysis. Different regions were analyzed to assure representative images.

\subsubsection{Differential Scanning Calorimetry (DSC)}

Nanoparticles energy storage capacity was measured by Differential Scanning Calorimetry (Jade DSC, Perkin Elmer) under nitrogen atmosphere at a heating and cooling rate of $10{ }^{\circ} \mathrm{C} / \mathrm{min}$. The heating scans were from $-10{ }^{\circ} \mathrm{C}$ to $30^{\circ} \mathrm{C}$, and the cooling scans were performed in the same range. The phase change latent heat $\left(\Delta H_{m}\right)$ and the melting temperature $\left(T_{m}\right)$ were recorded from the second heating ramp.

\subsubsection{PCM content in the nanoparticles}

The PCM (n-hexadecane) content in the nanoparticles was calculated based on the enthalpy value of pure n-hexadecane by using the following Equation $1^{[4,13,18]}$ :

$$
P C M \text { content in nanoparticles }(w t \%)=\frac{\Delta H_{n P C M}}{\Delta H_{P C M}} .100
$$

where $\triangle H_{n P C M}$ is the enthalpy of melting for the nanoencapsulated $\operatorname{PCM}(\mathrm{J} / \mathrm{g})$ and $\Delta H_{P C M}$ is the enthalpy of melting for the pure $\mathrm{n}$-hexadecane $(\mathrm{J} / \mathrm{g})$.

\subsubsection{Gel permeation chromatography (GPC)}

Molecular weight distributions were determined by gel permeation chromatography. The analyses were conducted in a high-performance liquid chromatography instrument (HPLC, model LC 20-A, Shimadzu) equipped with a PLgel MiniMIX-C (PL1510-1500) guard column, and two in series PLgel MiniMIX-C (PL1510-5500) HPLC columns, from
Agilent. Tetrahydrofuran (THF) was used as eluent (flow rate of $0.3 \mathrm{~mL} / \mathrm{min}$ ) at $40^{\circ} \mathrm{C}$. The calibration was performed using polystyrene standards with molecular weights ranging from $580 \mathrm{~g} / \mathrm{mol}$ to $9.225 \times 10^{6} \mathrm{~g} / \mathrm{mol}$.

\section{Results and Discussions}

Polystyrene nanoparticles with different $n$-hexadecane contents were prepared by miniemulsion polymerization using the ionic liquid $\mathrm{BMI} . \mathrm{Fe}_{2} \mathrm{Cl}_{7}$ as catalyst. In all cases, highly stable latexes containing PCM nanoparticles have been obtained, and no phase separation was observed. Monomer conversion was higher than $80 \%$ in all cases studied.

Dynamic light scattering (DLS) was used to analyze droplet (dd) and particle (dp) sizes of the miniemulsion and the latex, respectively. Table 1 summarizes the droplet (dd) and particle (dp) sizes before and after polymerization, respectively, and their dispersities (PdI).

PCM nanoparticles synthesized with different amounts of $\mathrm{n}$-hexadecane ranged from $138 \mathrm{~nm}$ to $158 \mathrm{~nm}$. According to the sizes obtained from droplets (miniemulsion, before polymerization) and polymeric particles (latex, after polymerization), droplet nucleation was the main nucleation mechanism and droplets/particles remained stable during polymerization. The miniemulsions produced with a higher amount of n-hexadecane had a slightly bigger droplet size. Increasing the amount of HD from $5 \mathrm{wt} \%$ to $50 \mathrm{wt} \%$, droplet size increased from $131 \mathrm{~nm}$ (nPCM-5) to $168 \mathrm{~nm}$ (nPCM-50). This behavior was also observed by Shirin-Abadi et al. ${ }^{[28]}$ in the synthesis of poly(methyl methacrylate) nanocapsules containing n-hexadecane as PCM. They suggested that this behavior may be due to the decreasing of the contribution of the surfactant molecules per particle when the HD amount is increased using the same amount of surfactant. However, added to the contribution of the surfactant molecules per particle there is the viscosity effect. An increase in the amount of HD increases the viscosity of the organic phase, and since the ultrasonication conditions were kept constant for all HD contents used, i.e. the same amount of energy was supplied to the system to break the organic phase into smaller droplets, the higher viscosity may have led to the increase in the droplet sizes. In the systems containing amounts of HD greater than $5 \mathrm{wt} \%$ it was observed a decrease in the difference between droplet and particle size compared to the system containing $5 \mathrm{wt} \%$ (nPCM-5). The narrow particle size distributions, Figure 1, also suggest that the main nucleation mechanism was the droplet nucleation and that secondary nucleation (homogeneous or micellar nucleation) did not take place.

Table 1. Effect of the n-hexadecane (HD) content on the droplet size (dd) and particle size (dp) and their dispersities (PdI). (Average values refer to duplicate reactions).

\begin{tabular}{ccccc}
\hline Entry & HD/Sty & dd (nm) & PdI (-) & dp (nm) \\
\hline nPCM-5 & $5 / 95$ & $131.2 \pm 8.8$ & $0.10 \pm 0.01$ & $158.4 \pm 2.4$ \\
nPCM-20 & $20 / 80$ & $138.4 \pm 4.8$ & $0.10 \pm 0.01$ & $137.5 \pm 2.4$ \\
nPCM-30 & $30 / 70$ & $141.4 \pm 4.8$ & $0.10 \pm 0.01$ & $142.3 \pm 3.9$ \\
nPCM-35 & $35 / 65$ & $148.9 \pm 1.3$ & $0.11 \pm 0.01$ & $140.6 \pm 2.1$ \\
nPCM-40 & $40 / 60$ & $154.4 \pm 1.1$ & $0.09 \pm 0.01$ & $0.04 \pm 0.02$ \\
nPCM-50 & $50 / 50$ & $168.0 \pm 9.2$ & $0.16 \pm 0.06$ & $0.06 \pm 0.02$ \\
\hline
\end{tabular}


The morphology of nanoparticles containing the phase change material (nPCM) was observed by Transmission Electron Microscopy (TEM). The micrographs of the $\mathrm{nPCM}$ with $50 \mathrm{wt} \%$ of $\mathrm{HD}$ in relation to the organic phase (sample nPCM-50), Figure 2, show that the nanoparticles obtained have a spherical shape with a fairly uniform size distribution.

From the TEM images it is not possible to identify a capsule morphology (lighter core of HD surrounded by a darker PS shell), but according to Tiarks et al. ${ }^{[29]}$ for the same system HD/Sty due to the similarity of the particle sizes obtained by DLS $(150.6 \mathrm{~nm})$ and TEM, it is deduced that the HD is located within the particles, presumably in a spongelike morphology.

Molecular weight distributions were obtained using gel permeation chromatography. The weight-average molecular weight $(\mathrm{Mw})$ and the molecular-weight dispersity $(\nexists)$ of the polystyrene nanoparticles are summarized in Table 2.

GPC data shows that the cationic miniemulsion polymerization of styrene catalyzed by the ionic liquid BMI. $\mathrm{Fe}_{2} \mathrm{Cl}_{7}$ provided the synthesis of high molecular weight polystyrene nanoparticles. Under similar experimental conditions of the $\mathrm{nPCM}-5$ system, $5 \mathrm{wt} \%$ of HD in relation to the organic phase, 1:1000 molar ratio of IL:Sty and at $85^{\circ} \mathrm{C}$, Alves et al. ${ }^{[26]}$ obtained average viscosity molar mass $(\mathrm{Mv})$ up to $2150 \mathrm{kDa}$, at conversions slightly higher than $80 \%$. It is worth mentioning that the high amount of $\mathrm{HD}$ in the system (nPCM-20 to nPCM-50) did not affect the high molecular weights obtained which were similar to those achieved when the HD was used only as costabilizer (nPCM-5).

The molecular-weight dispersities $(\nexists)$ denote broad molecular weight distributions. Rodrigues et al. ${ }^{[27]}$ by on-line direct infusion electrospray mass spectrometry (ESI-MS(/MS)) detected three active catalytic species in the cationic polymerization of styrene catalyzed by the ionic liquid BMI. $\mathrm{Fe}_{2} \mathrm{Cl}_{7}$. These three active catalytic species: (i) the chloronium cation (derived from styrene), (ii) the chloronium cation associated with $\left[\mathrm{FeCl}_{4}\right]^{-}$and (iii) the chloronium cation associated with $\mathrm{Cl}^{-}$have different reactivities. The presence of active catalytic species with different reactivities elucidates the broad molecular weight distributions obtained.

Thermal properties of the $\mathrm{nPCM}$ were measured by DSC. The DSC curves of the n-hexadecane nanoencapsulated in high molecular weight polystyrene polymeric matrix synthesized using different HD weight ratios in relation to the organic phase are presented in Figure 3 and summarized in Table 3. Also measured by DSC, the heat storage capacity of pure $\mathrm{n}$-hexadecane, defined as the heat storage of melting $\left(\Delta H_{\mathrm{m}}\right)$, and its melting temperature $\left(T_{\mathrm{m}}\right)$ were $290.35 \mathrm{~J} / \mathrm{g}$ and $16.4{ }^{\circ} \mathrm{C}$, respectively.

The enthalpy of melting for the nanoencapsulated PCM ranged from $19 \mathrm{~J} / \mathrm{g}$ to $72 \mathrm{~J} / \mathrm{g}$, increasing as the amount of $\mathrm{HD}$ in the system also increased. By increasing the HD content in the organic phase, the values of melting enthalpy also increased due to the higher contribution of HD during the encapsulation process. Similar behavior was described by Shirin-Abadi et al. ${ }^{[28]}$ in the in situ encapsulation of n-hexadecane with poly(methyl methacrylate) shell by

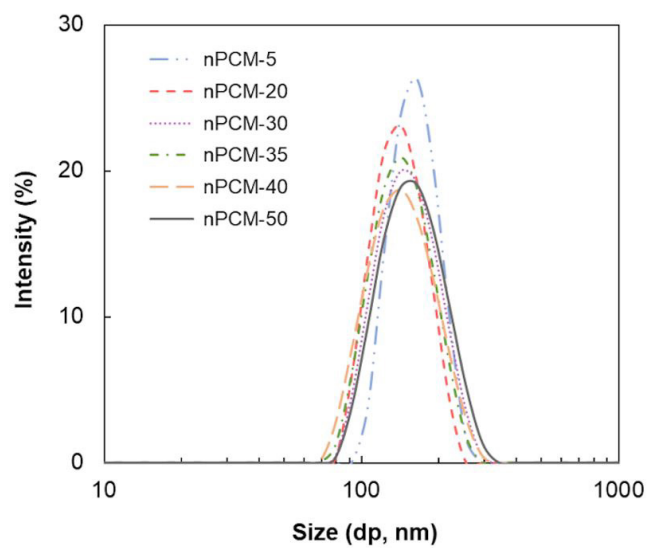

Figure 1. Particle size distribution of polymeric latexes synthesized with different $\mathrm{HD} /$ Sty weight ratios.
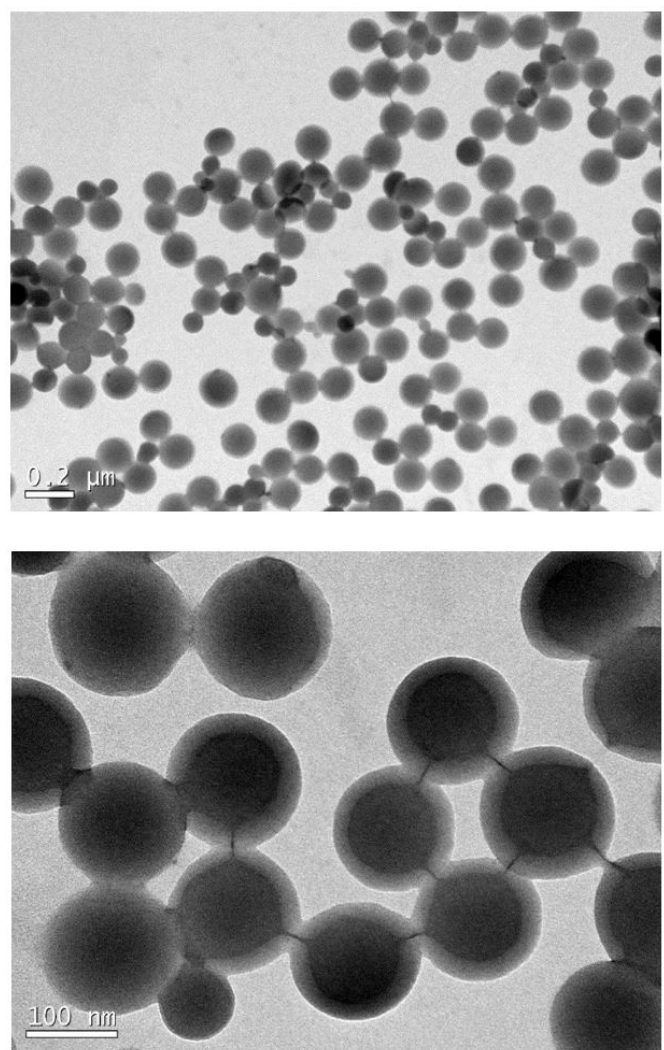

Figure 2. TEM micrographs of the polystyrene nanoparticles obtained in miniemulsion polymerization at $85^{\circ} \mathrm{C}$ using an IL:Sty molar ratio of 1:1000 and $50 \mathrm{wt} \%$ of $\mathrm{HD}$ in relation to the organic phase, sample nPCM-50 (Table 1).

Table 2. Molecular weight of nPCM synthesized with different $\mathrm{HD}$ weight ratios in relation to the organic phase. (Average values refer to duplicate reactions).

\begin{tabular}{cccc}
\hline Entry & HD/Sty & Mw (kDa) & $\boldsymbol{D}(-)$ \\
\hline nPCM-5 & $5 / 95$ & $1771 \pm 24$ & $2.1 \pm 0.1$ \\
nPCM-20 & $20 / 80$ & $1572 \pm 43$ & $2.7 \pm 0.1$ \\
nPCM-30 & $30 / 70$ & $1370 \pm 19$ & $2.9 \pm 0.1$ \\
nPCM-35 & $35 / 65$ & $1238 \pm 20$ & $3.0 \pm 0.1$ \\
nPCM-40 & $40 / 60$ & $1456 \pm 26$ & $2.7 \pm 0.1$ \\
nPCM-50 & $50 / 50$ & $1693 \pm 228$ & $2.2 \pm 0.1$ \\
\hline
\end{tabular}




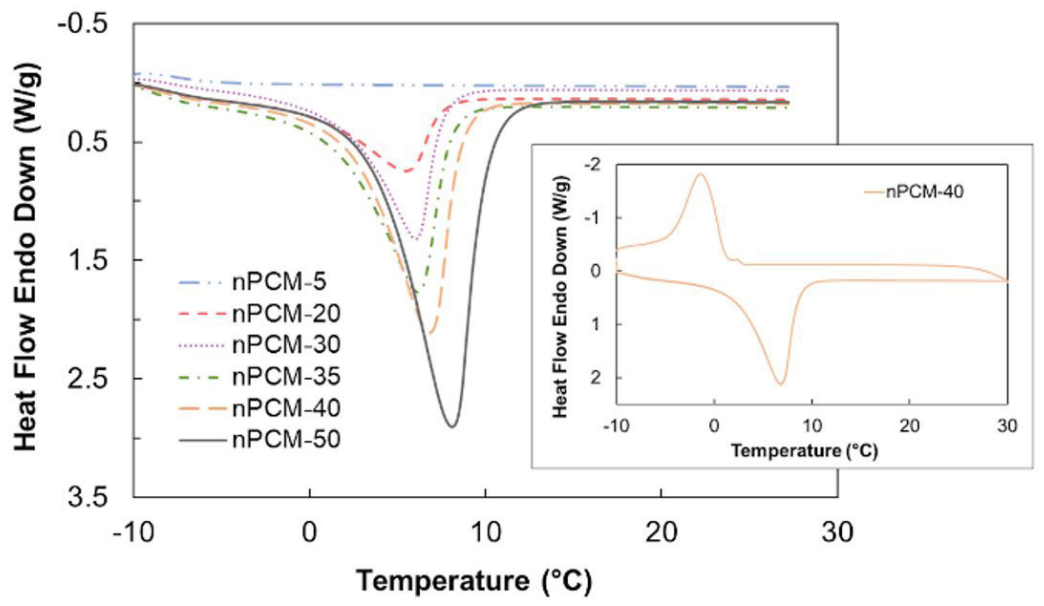

Figure 3. DSC curves of nPCM synthesized with different HD/Sty weight ratios.

Table 3. Thermal properties of nPCM synthesized with different HD/Sty weight ratios. (Average values refer to duplicate reactions).

\begin{tabular}{ccccc}
\hline Sample & HD/Sty & $\boldsymbol{\Delta H}_{\mathbf{m}}(\mathbf{J} / \mathbf{g})$ & $T_{\mathbf{m}}\left({ }^{\circ} \mathbf{C}\right)$ & PCM content $(\mathbf{w t} \%)$ \\
\hline nPCM-5 & $5 / 95$ & - & - & - \\
nPCM-20 & $20 / 80$ & $18.77 \pm 0.78$ & $5.67 \pm 0.28$ & 6.46 \\
nPCM-30 & $30 / 70$ & $27.30 \pm 7.44$ & $6.01 \pm 0.01$ & 9.40 \\
nPCM-35 & $35 / 65$ & $43.58 \pm 0.07$ & $6.60 \pm 0.67$ & 15.01 \\
nPCM-40 & $40 / 60$ & $50.85 \pm 1.30$ & $6.83 \pm 0.12$ & 17.51 \\
nPCM-50 & $50 / 50$ & $71.77 \pm 5.01$ & $7.92 \pm 0.33$ & 24.72 \\
\hline
\end{tabular}

miniemulsion polymerization. They obtained enthalpy of melting for the nanoencapsulated PCM ranging from $67 \mathrm{~J} / \mathrm{g}$ to $86 \mathrm{~J} / \mathrm{g}$ for HD:MMA weight ratios ranging from $1: 3$ to $3: 1$. Final PCM content in the nanoparticles ranged from $6.46 \mathrm{wt} \%$ to $24.72 \mathrm{wt} \%$ for an increase in $\mathrm{HD}$ content from $20 \mathrm{wt} \%$ (nPCM-20) to $50 \mathrm{wt} \%$ (nPCM-50) in relation to the organic phase.

The nPCM- 5 that was synthesized with $5 \mathrm{wt} \%$ of HD did not exhibit enthalpy of melting. This absence of enthalpy denotes an unwanted plasticizing effect of the n-hexadecane on nanoparticles. By acting as plasticizer part of the HD is incorporated into the polymer matrix, reducing the amount of free HD that can act as PCM. The plasticizing effect significantly decreases the glass transition temperature of the polymer. To evaluate the plasticizing effect, the glass transition temperature of samples nPCM-5 and nPCM-50 was determined by DSC under nitrogen atmosphere at a heat rate of $10^{\circ} \mathrm{C} / \mathrm{min}$ in a temperature range from $-10^{\circ} \mathrm{C}$ to $140{ }^{\circ} \mathrm{C}$. The glass transition temperature $\left(T_{g}\right)$ was recorded from the second heating ramp. The $T_{g}$ of sample nPCM-5 was $77.3^{\circ} \mathrm{C}$, while the $T_{g}$ of sample nPCM-50 dropped to $54.5^{\circ} \mathrm{C}$.

The phase change temperature of the n-hexadecane contained in the nanoparticles was below the melting temperature of pure $\mathrm{n}$-hexadecane $\left(16.4{ }^{\circ} \mathrm{C}\right)$ and ranged from $5.67{ }^{\circ} \mathrm{C}$ to $7.92{ }^{\circ} \mathrm{C}$ when the $\mathrm{HD}$ content was increased from $20 \mathrm{wt} \%$ (nPCM-5) to $50 \mathrm{wt} \%$ (nPCM-50). Chen et al. ${ }^{[18,19]}$. also observed a reduction in the phase change temperature of the $n$-dodecanol contained in the nanocapsules in relation to the pure $\mathrm{n}$-dodecanol $\left(T_{\mathrm{m}}=27^{\circ} \mathrm{C}\right)$ used as PCM. The nanocapsules containing n-dodecanol as the core and poly(methyl methacrylate) (PMMA) $)^{[18]}$ or styrene-butyl acrylate copolymer ${ }^{[19]}$ as the shell showed a
Table 4. Thermal properties of the nPCM synthesized with $40 \mathrm{wt} \%$ of $\mathrm{HD}$ in relation to the organic phase (nPCM-40) after different numbers of thermal cycles.

\begin{tabular}{ccc}
\hline Thermal cycles & $\boldsymbol{\Delta H}_{\mathrm{m}}(\mathbf{J} / \mathbf{g})$ & $T_{\mathrm{m}}\left({ }^{\circ} \mathbf{C}\right)$ \\
\hline 0 & 49.66 & 6.91 \\
20 & 49.84 & 6.92 \\
40 & 50.34 & 6.92 \\
60 & 50.11 & 6.92 \\
80 & 50.15 & 6.92 \\
100 & 50.16 & 6.91 \\
\hline
\end{tabular}

phase change temperature of $18.2^{\circ} \mathrm{C}$ and $18.4{ }^{\circ} \mathrm{C}$, respectively. Fang et al. ${ }^{[2]}$ reported a slight difference in the melting points between the core material and the nanocapsules. The melting temperature for pure n-tetradecane (Tet) used as PCM was $6.94^{\circ} \mathrm{C}$, whereas the change phase temperature for the Tet/PS nanocapsules was $4.04^{\circ} \mathrm{C}$. They suggested that this difference may be related to the slower heat conduction rate of the polymer shell that delays the phase change process of the nanocapsules.

The thermal reversibility of the $\mathrm{NPCM}$ was investigated until 100 thermal cycles (heat/cooling) carried out by using DSC. The thermal properties of nPCM-40 after different numbers of thermal cycles are summarized in Table 4.

The phase change latent heat of sample nPCM- 40 remained virtually constant after 100 cycles, ranging from 49 to $50 \mathrm{~J} / \mathrm{g}$. Thus, the nanoencapsulated n-hexadecane nanoparticles show thermal reversibility after 100 thermal cycles. The melting temperature also remained constant at $6.9^{\circ} \mathrm{C}$. 


\section{Conclusions}

The cationic miniemulsion polymerization of styrene catalyzed by the ionic liquid $\mathrm{BMI} . \mathrm{Fe}_{2} \mathrm{Cl}_{7}$ has proved to be a convenient one-step encapsulation technique to encapsulate a phase change material into high molecular weight polymeric nanoparticles. Particle size analyses (DLS) and TEM micrographs indicated that polymeric particles are spherical, with narrow size distribution and particle size ranging from $138 \mathrm{~nm}$ to $158 \mathrm{~nm}$. In addition, these analyses also denoted that the HD is located within the particles, presumably in a spongelike morphology.

The presence of HD in the nanoparticles was confirmed by the DSC analyses that have shown the enthalpy of melting for the nanoencapsulated n-hexadecane. The enthalpy of melting increased from $19 \mathrm{~J} / \mathrm{g}$ to $72 \mathrm{~J} / \mathrm{g}$, as the content of $\mathrm{n}$-hexadecane used increased from $20 \mathrm{wt} \%$ to $50 \mathrm{wt} \%$ in relation to the organic phase. DSC analyses also supported the thermal reversibility of nanoparticles after 100 thermal cycles. DLS, TEM, and DSC results also indicated that the HD acted simultaneously as hydrophobic agent and as phase change material.

As expected, the low ionic liquid concentration (1:1000 molar ratio of IL:Sty) used as catalyst leaded to high molecular weights, up to $1800 \mathrm{kDa}$, even in the presence of high amounts of HD. These high molecular weights may have contributed positively to the thermal behavior of the nanoparticles.

\section{Acknowledgements}

The authors thank the financial support from CAPES - Coordenação de Aperfeiçoamento de Pessoal de Nível Superior, Finance Code 001 and CNPq - Conselho Nacional de Desenvolvimento Científico e Tecnológico. The authors also thank the Central Laboratory of Electron Microscopy - LCME and the Analysis Center of Chemical Engineering and Food Engineering Department from the Federal University of Santa Catarina for the TEM and DSC analyses, respectively.

\section{References}

1. Su, W., Darkwa, J., \& Kokogiannakis, G. (2015). Review of solid-liquid phase change materials and their encapsulation technologies. Renewable \& Sustainable Energy Reviews, 48, 373-391. http://dx.doi.org/10.1016/j.rser.2015.04.044.

2. Fang, Y., Yu, H., Wan, W., Gao, X., \& Zhang, Z. (2013). Preparation and thermal performance of polystyrene/n-tetradecane composite nanoencapsulated cold energy storage phase change materials. Energy Conversion and Management, 76, 430-436. http://dx.doi.org/10.1016/j.enconman.2013.07.060.

3. Khadiran, T., Hussein, M. Z., Zainal, Z., \& Rusli, R. (2015). Encapsulation techniques for organic phase change materials as thermal energy storage medium: A review. Solar Energy Materials and Solar Cells, 143, 78-98. http://dx.doi.org/10.1016/j. solmat.2015.06.039.

4. Rezvanpour, M., Hasanzadeh, M., Azizi, D., Rezvanpour, A., \& Alizadeh, M. (2018). Synthesis and characterization of micro-nanoencapsulated n-eicosane with PMMA shell as novel phase change materials for thermal energy storage. Materials Chemistry and Physics, 215, 299-304. http://dx.doi. org/10.1016/j.matchemphys.2018.05.044.
5. Landfester, K. (2003). Miniemulsions for nanoparticle synthesis. Topics in Current Chemistry, 227, 75-123. http:// dx.doi.org/10.1007/3-540-36412-9_4.

6. Landfester, K. (2009). Miniemulsion polymerization and the structure of polymer and hybrid nanoparticles. Angewandte Chemie International Edition, 48(25), 4488-4507. http://dx.doi. org/10.1002/anie.200900723. PMid:19455531.

7. Ouzineb, K., Lord, C., Lesauze, N., Graillat, C., Tanguy, P. A., \& McKenna, T. (2006). Homogenisation devices for the production of miniemulsions. Chemical Engineering Science, 61(9), 2994-3000. http://dx.doi.org/10.1016/j.ces.2005.10.065.

8. Tang, P. L., Sudol, E. D., Silebi, C. A., \& El-Aasser, M. S. (1991). Miniemulsion polymerization - A comparative study of preparative variables. Journal of Applied Polymer Science, 43(6), 1059-1066. http://dx.doi.org/10.1002/app.1991.070430604.

9. Agner, T., Zimmermann, A., Di Luccio, M., Araújo, P. H. H., \& Sayer, C. (2017). Monomer-in-water miniemulsions by membrane emulsification. Chemical Engineering and Processing - Process Intensification, 120, 251-257. http:// dx.doi.org/10.1016/j.cep.2017.07.016.

10. do Amaral, M., \& Asua, J. M. (2004). Synthesis of large, high-solid-content latexes by miniemulsion polymerization. Journal of Polymer Science. Part A, Polymer Chemistry, 42(17), 4222-4227. http://dx.doi.org/10.1002/pola.20287.

11. Farzi, G., Bourgeat-Lami, E., \& McKenna, T. F. L. (2009). Miniemulsions using static mixers: A feasibility study using simple in-line static mixers. Journal of Applied Polymer Science, 114(6), 3875-3881. http://dx.doi.org/10.1002/app.30343.

12. Luo, Y., \& Zhou, X. (2004). Nanoencapsulation of a hydrophobic compound by a miniemulsion polymerization process. Journal of Polymer Science. Part A, Polymer Chemistry, 42(9), 21452154. http://dx.doi.org/10.1002/pola.20065.

13. de Cortazar, M. G., \& Rodríguez, R. (2013). Thermal storage nanocapsules by miniemulsion polymerization. Journal of Applied Polymer Science, 127(6), 5059-5064. http://dx.doi. org/10.1002/app.38124.

14. Fang, Y., Kuang, S., Gao, X., \& Zhang, Z. (2008). Preparation and characterization of novel nanoencapsulated phase change materials. Energy Conversion and Management, 49(12), 37043707. http://dx.doi.org/10.1016/j.enconman.2008.06.027.

15. Fang, Y., Kuang, S., Gao, X., \& Zhang, Z. (2009). Preparation of nanoencapsulated phase change material as latent functionally thermal fluid. Journal of Physics D: Applied Physics, 42(3), 035407. http://dx.doi.org/10.1088/0022-3727/42/3/035407.

16. Fang, Y., Liu, X., Liang, X., Liu, H., Gao, X., \& Zhang, Z. (2014). Ultrasonic synthesis and characterization of polystyrene/ndotriacontane composite nanoencapsulated phase change material for thermal energy storage. Applied Energy, 132, 551-556. http://dx.doi.org/10.1016/j.apenergy.2014.06.056.

17. Fuensanta, M., Paiphansiri, U., Romero-Sánchez, M. D., Guillem, C., López-Buendía, Á. M., \& Landfester, K. (2013). Thermal properties of a novel nanoencapsulated phase change material for thermal energy storage. Thermochimica Acta, 565, 95-101. http://dx.doi.org/10.1016/j.tca.2013.04.028.

18. Chen, Z.-H., Yu, F., Zeng, X.-R., \& Zhang, Z.-G. (2012). Preparation, characterization and thermal properties of nanocapsules containing phase change material $n$-dodecanol by miniemulsion polymerization with polymerizable emulsifier. Applied Energy, 91(1), 7-12. http://dx.doi.org/10.1016/j. apenergy.2011.08.041

19. Chen, C., Chen, Z., Zeng, X., Fang, X., \& Zhang, Z. (2012). Fabrication and characterization of nanocapsules containing n-dodecanol by miniemulsion polymerization using interfacial redox initiation. Colloid \& Polymer Science, 290(4), 307-314. http://dx.doi.org/10.1007/s00396-011-2545-2. 
20. Barrère, M., Ganachaud, F., Bendejacq, D., Dourges, M.-A., Maitre, C., \& Hémery, P. (2001). Anionic polymerization of octamethylcyclotetrasiloxane in miniemulsion II. Molar mass analyses and mechanism scheme. Polymer, 42(17), 7239-7246. http://dx.doi.org/10.1016/S00323861(01)00207-5.

21. Barrère, M., Maitre, C., Dourges, M. A., \& Hémery, P. (2001). Anionic polymerization of 1,3,5-tris(trifluoropropylmethyl) cyclotrisiloxane $\left(\mathrm{F}_{3}\right)$ in miniemulsion. Macromolecules, 34(21), 7276-7280. http://dx.doi.org/10.1021/ma010559z.

22. Crespy, D., \& Landfester, K. (2005). Anionic polymerization of $\varepsilon$-caprolactam in miniemulsion: Synthesis and characterization of polyamide-6 nanoparticles. Macromolecules, 38(16), 68826887. http://dx.doi.org/10.1021/ma050616e.

23. Cauvin, S., Sadoun, A., Santos, R., Belleney, J., Ganachaud, F., \& Hemery, P. (2002). Cationic polymerization of p-methoxystyrene in miniemulsion. Macromolecules, 35(21), 7919-7927. http:// dx.doi.org/10.1021/ma0202890.

24. Touchard, V., Graillat, C., Boisson, C., D’Agosto, F., \& Spitz, R. (2004). Use of a Lewis acid surfactant combined catalyst in cationic polymerization in miniemulsion: Apparent and hidden initiators. Macromolecules, 37(9), 3136-3142. http:// dx.doi.org/10.1021/ma0355352.

25. Cauvin, S., Ganachaud, F., Moreau, M., \& Hémery, P. (2005). High molar mass polymers by cationic polymerisation in emulsion and miniemulsion. Chemical Communications, 1(21), 2713 2715. http://dx.doi.org/10.1039/b501489a. PMid:15917929.

26. Alves, R. C., Agner, T., Rodrigues, T. S., Machado, F., Neto, B. A. D., Costa, C., Araújo, P. H. H., \& Sayer, C. (2018). Cationic miniemulsion polymerization of styrene mediated by imidazolium based ionic liquid. European Polymer Journal, 104, 51-56. http://dx.doi.org/10.1016/j.eurpolymj.2018.04.035.

27. Rodrigues, T. S., Machado, F., Lalli, P. M., Eberlin, M. N., $\&$ Neto, B. A. D. (2015). Styrene polymerization efficiently catalyzed by iron-containing imidazolium-based ionic liquids: Reaction mechanism and enhanced ionic liquid effect. Catalysis Communications, 63, 66-73. http://dx.doi.org/10.1016/j. catcom.2014.11.002.

28. Shirin-Abadi, A. R., Mahdavian, A. R., \& Khoee, S. (2011). New approach for the elucidation of PCM nanocapsules through miniemulsion polymerization with an acrylic shell. Macromolecules, 44(18), 7405-7414. http://dx.doi.org/10.1021/ ma201509d.

29. Tiarks, F., Landfester, K., \& Antonietti, M. (2001). Preparation of polymeric nanocapsules by miniemulsion polymerization. Langmuir, 17(3), 908-918. http://dx.doi.org/10.1021/la001276n.

Received: Feb. 10, 2020

Revised: May 23, 2020

Accepted: June 15, 2020 